\title{
Application of Homotopy Perturbation Method with an Auxiliary Term for Nonlinear Dropping Equations Arisen in Polymer Packaging System
}

\author{
Xiang Hong, 1 Jun Wang, ${ }^{1,2}$ and Li-xin $\mathrm{Lu}^{1,2}$ \\ ${ }^{1}$ Department of Packaging Engineering, Jiangnan University, Wuxi 214122, China \\ ${ }^{2}$ Key Laboratory of Food Packaging Techniques and Safety of China National Packaging Corporation, Wuxi 214122, China \\ Correspondence should be addressed to Jun Wang; wangj_1982@jiangnan.edu.cn and Li-xin Lu; lulx@jiangnan.edu.cn
}

Received 6 February 2013; Revised 28 March 2013; Accepted 23 April 2013

Academic Editor: Dong Sun Lee

Copyright (C) 2013 Xiang Hong et al. This is an open access article distributed under the Creative Commons Attribution License, which permits unrestricted use, distribution, and reproduction in any medium, provided the original work is properly cited.

The homotopy perturbation method (HPM) with an auxiliary term was applied to obtain approximate analytical solutions of polymer cushioning packaging system. The second-order solution of the equation of motion was obtained and compared with the numerical simulation solution solved by the Runge-Kutta algorithm. The results showed the high accuracy of this modified HPM with convenient calculation.

\section{Introduction}

Dropping is an unavoidable situation for a packaged product while delivered, which is investigated by many researchers [1-3]. In most cases, the constitutive models of cushioning package materials are strong nonlinear.

Various kinds of nonlinear oscillation problems exist in the engineering field, which are usually difficult to be solved analytically. However, the analytical solution is more significant for the further intensive study. Among the methods for analytical solution, the perturbation method [4] is one of the most well-known approaches, and it is based on the existence of small parameters which are not commonly existed in many nonlinear problems. Besides, in order to avoid some restrictions of perturbation method, some other methods are developed, including the variational iteration method (VIM) [5], the homotopy analysis method (HAM) [6], He's max-min method, and the homotopy perturbation method (HPM) [711]. HPM is an analytical method providing an alternative approach to introducing an expanding parameter and applied to many areas of science and engineering [12-17]. Nawaz [18] studied nonlinear boundary value problems for fourth-order fractional integrodifferential equations using both VIM and HPM, and the comparison results showed that both methods were very effective and convenient to solve this problem.
Noor [19] modified the HPM with an auxiliary term which makes the HPM more flexible. In recent studies, He [20] summarized the modification of the HPM by introducing an auxiliary term in the homotopy equation, and Duffing equation was used as an example to illustrate the solution procedure.

Polymer foams, especially expanded polystyrene (EPS), are widely used for cushion or protective inner packaging, and the governing equations [21] can be expressed as

$$
\begin{gathered}
m \ddot{x}+\beta_{3} \tanh \left(\beta_{1} x\right)+\beta_{4} \tan \left(\beta_{2} x\right) \\
+\beta_{5} \tan ^{3}\left(\beta_{2} x\right)=0, \quad x(0)=0, \\
\dot{x}(0)=\sqrt{2 g h},
\end{gathered}
$$

where $x$ denotes the displacement of the product while dropping, $\mathrm{m}$; the coefficient $m$ denotes the mass of the packaged product, $\mathrm{kg} ; \beta_{1}$ and $\beta_{2}$ are the displacement impendence, $\mathrm{m}^{-1} ; \beta_{3}, \beta_{4}$, and $\beta_{5}$ are the elasticity, $\mathrm{N}$, and $\beta_{\mathrm{i}}$ denote, respectively, the characteristic constants of polymer foams which could be obtained by compression test; $g$ is the acceleration of gravity, $\mathrm{m} / \mathrm{s}^{-2}$; and $h$ is the dropping height, $\mathrm{m}$. The polymer packaging system can be shown in Figure 1 . 


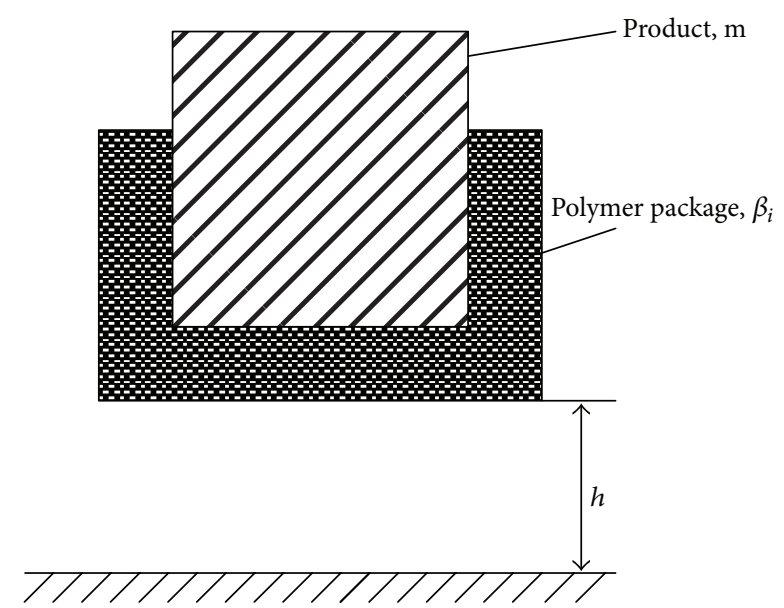

FIGURE 1: Polymer packaging system.

By introducing these parameters: $T_{0}=\sqrt{m / \beta_{1} \beta_{3}}, L=$ $1 / \beta_{1}, \lambda_{1}=\beta_{2} / \beta_{1}, \lambda_{2}=\beta_{4} / \beta_{3}$, and $\lambda_{3}=\beta_{5} / \beta_{3}$, and letting $X=x / L, \tau=t / T_{0}$, (1) can be written in the following nondimensional forms:

$$
\begin{array}{r}
\ddot{X}+\tanh X+\lambda_{2} \tan \left(\lambda_{1} X\right)+\lambda_{3} \tan ^{3}\left(\lambda_{1} X\right)=0, \\
X(0)=0, \\
\dot{X}(0)=V=\frac{T_{0}}{L} \sqrt{2 g h}=\sqrt{\frac{2 \beta_{1} m g h}{\beta_{3}}} .
\end{array}
$$

This paper investigated for the first time the applicability and the validity of the modified HPM for EPS polymer cushioning packaging system. Besides, in order to show the accuracy of this method, some specific parameters were used in the constitutive equation based on real situation, and solutions of the modified HPM and Runge-Kutta method were compared.

\section{Homotopy Perturbation Method with an Auxiliary Term}

Considering a general nonlinear equation

$$
L u+N u=0,
$$

where $L$ and $N$ are the linear operator and nonlinear operator, respectively.

According to the classic HPM, the homotopy equation can be constructed as

$$
\widetilde{L} u+p(L u-\widetilde{L} u+N u)=0
$$

where $\widetilde{L}$, constructed based on the model property, is a linear operator and $\widetilde{L} u=0$ can depict the solution property. With the increase of the embedding parameter $p$ from 0 to $1,(4)$ will transform from $\widetilde{L} u=0$ to the original one.

According to He's recent study [20], the homotopy equation can be constructed with an auxiliary term as

$$
\widetilde{L} u+p(L u-\widetilde{L} u+N u)+\alpha p(1-p) u=0,
$$

where $\alpha$ is the auxiliary parameter. It is obvious that when $\alpha=0$, (5) is equivalent to the classical equation (4). And $\alpha$ can be determined in the iteration procedure.

The solutions of both (4) and (5) can be expanded into a series in $p$ :

$$
u=u_{0}+p u_{1}+p^{2} u_{2}+\cdots
$$

\section{Nonlinear Polymer Packaging System}

By the Taylor series, (2) can be expanded by Taylor series as follows to simplify the calculation:

$$
\begin{aligned}
\ddot{X} & +X\left(\lambda_{1} \lambda_{2}+1\right) \\
& +X^{3}\left(\frac{\lambda_{1}^{3} \lambda_{2}}{3}+\lambda_{1}^{3} \lambda_{3}-\frac{1}{3}\right) \\
& +X^{5}\left(\frac{2 \lambda_{1}^{5} \lambda_{2}}{15}+\lambda_{1}^{5} \lambda_{3}+\frac{2}{15}\right)=0 .
\end{aligned}
$$

According to [16], the homotopy equation with an auxiliary term can be constructed as

$$
\begin{aligned}
\ddot{X}+\Omega^{2} X+p[ & \left(\lambda_{1} \lambda_{2}+1-\Omega^{2}\right) X \\
& +\left(\frac{\lambda_{1}^{3} \lambda_{2}}{3}+\lambda_{1}^{3} \lambda_{3}-\frac{1}{3}\right) X^{3} \\
& \left.+\left(\frac{2 \lambda_{1}^{5} \lambda_{2}}{15}+\lambda_{1}^{5} \lambda_{3}+\frac{2}{15}\right) X^{5}\right] \\
+\alpha p(1-p) X & =0 .
\end{aligned}
$$

According to the classical perturbation method, the iteration equations can be constructed as

$$
\begin{aligned}
\ddot{X}_{0}+\Omega^{2} X_{0} & =0, \quad X_{0}(0)=0, \quad \dot{X}_{0}(0)=V, \\
\ddot{X}_{1} & +\Omega^{2} X_{1}+X_{0}\left(\lambda_{1} \lambda_{2}+1-\Omega^{2}+\alpha\right) \\
& +X_{0}^{3}\left(\frac{\lambda_{1}^{3} \lambda_{2}}{3}+\lambda_{1}^{3} \lambda_{3}-\frac{1}{3}\right) \\
& +X_{0}^{5}\left(\frac{2 \lambda_{1}^{5} \lambda_{2}}{15}+\lambda_{1}^{5} \lambda_{3}+\frac{2}{15}\right)=0 \\
\ddot{X}_{2} & +\Omega^{2} X_{2}+X_{1}\left(\lambda_{1} \lambda_{2}+1-\Omega^{2}+\alpha\right) \\
& +3 X_{0}^{2} X_{1}\left(\frac{\lambda_{1}^{3} \lambda_{2}}{3}+\lambda_{1}^{3} \lambda_{3}-\frac{1}{3}\right) \\
& +5 X_{0}^{4} X_{1}\left(\frac{2 \lambda_{1}^{5} \lambda_{2}}{15}+\lambda_{1}^{5} \lambda_{3}+\frac{2}{15}\right)=0 .
\end{aligned}
$$

Solve (9) and then obtain the initial approximate solution

$$
X_{0}=A \sin \Omega \tau, \quad A \Omega=V,
$$


where $A$ and $\Omega$ are, respectively, the displacement amplitude and the frequency, and can be further determined.

Substitute (12) into (10) and rewrite it as

$$
\ddot{X}_{1}+\Omega^{2} X_{1}+K_{1} \sin \Omega t+K_{2} \sin 3 \Omega t+K_{3} \sin 5 \Omega t=0,
$$

where

$$
\begin{aligned}
K_{1}= & \left(\lambda_{1} \lambda_{2}+1-\Omega^{2}+\alpha\right) A \\
& +\frac{3}{4}\left(\frac{\lambda_{1}^{3} \lambda_{2}}{3}+\lambda_{1}^{3} \lambda_{3}-\frac{1}{3}\right) A^{3} \\
& +\frac{5}{8}\left(\frac{2 \lambda_{1}^{5} \lambda_{2}}{15}+\lambda_{1}^{5} \lambda_{3}+\frac{2}{15}\right) A^{5}, \\
K_{2}= & -\frac{1}{4}\left(\frac{\lambda_{1}^{3} \lambda_{2}}{3}+\lambda_{1}^{3} \lambda_{3}-\frac{1}{3}\right) A^{3} \\
& -\frac{5}{16}\left(\frac{2 \lambda_{1}^{5} \lambda_{2}}{15}+\lambda_{1}^{5} \lambda_{3}+\frac{2}{15}\right) A^{5}, \\
K_{3}= & \frac{1}{16}\left(\frac{2 \lambda_{1}^{5} \lambda_{2}}{15}+\lambda_{1}^{5} \lambda_{3}+\frac{2}{15}\right) A^{5} .
\end{aligned}
$$

In order to eliminate the secular term, it must be satisfied that $K_{1}=0$. Thus,

$$
\begin{aligned}
\Omega^{2}= & \left(\lambda_{1} \lambda_{2}+1+\alpha\right) \\
& +\frac{3}{4}\left(\frac{\lambda_{1}^{3} \lambda_{2}}{3}+\lambda_{1}^{3} \lambda_{3}-\frac{1}{3}\right) A^{2} \\
& +\frac{5}{8}\left(\frac{2 \lambda_{1}^{5} \lambda_{2}}{15}+\lambda_{1}^{5} \lambda_{3}+\frac{2}{15}\right) A^{4} .
\end{aligned}
$$

A special solution of the ordinary differential equation (13) can be easily obtained as

$$
X_{1}=\frac{K_{2}}{8 \Omega^{2}} \sin 3 \Omega \tau+\frac{K_{3}}{24 \Omega^{2}} \sin 5 \Omega \tau .
$$

Thus, by substituting (16) into (11), the second-order iteration equation can be expressed as

$$
\begin{aligned}
\ddot{X}_{2} & +\Omega^{2} X_{2}+M_{1} \sin \Omega \tau+M_{2} \sin 3 \Omega \tau \\
& +M_{3} \sin 5 \Omega \tau+M_{4} \sin 7 \Omega \tau+M_{5} \sin 9 \Omega \tau=0,
\end{aligned}
$$

where

$$
\begin{aligned}
M_{1}= & \frac{19\left(2 \lambda_{1}^{5} \lambda_{2}+15 \lambda_{1}^{5} \lambda_{3}+2\right)^{2}}{69120 \Omega^{2}} A^{8} \\
& +\frac{\left(\lambda_{1}^{3} \lambda_{2}+3 \lambda_{1}^{3} \lambda_{3}-1\right)\left(2 \lambda_{1}^{5} \lambda_{2}+15 \lambda_{1}^{5} \lambda_{3}+2\right)}{576 \Omega^{2}} A^{6} \\
& +\frac{\left(\lambda_{1}^{3} \lambda_{2}+3 \lambda_{1}^{3} \lambda_{3}-1\right)^{2}}{384 \Omega^{2}} A^{4}-\alpha,
\end{aligned}
$$

$$
\begin{aligned}
M_{2}= & \frac{\left(2 \lambda_{1}^{5} \lambda_{2}+15 \lambda_{1}^{5} \lambda_{3}+2\right)\left(9 K_{2}-2 K_{3}\right)}{576 \Omega^{2}} A^{4} \\
& +\frac{\left(\lambda_{1}^{3} \lambda_{2}+3 \lambda_{1}^{3} \lambda_{3}-1\right)\left(6 K_{2}-K_{3}\right)}{96 \Omega^{2}} A^{2} \\
& -\frac{K_{2}\left(\Omega^{2}-\alpha-\lambda_{1} \lambda_{2}-1\right)}{8 \Omega^{2}}, \\
M_{3}= & -\frac{\left(2 \lambda_{1}^{5} \lambda_{2}+15 \lambda_{1}^{5} \lambda_{3}+2\right)\left(2 K_{2}-K_{3}\right)}{192 \Omega^{2}} A^{4} \\
& -\frac{\left(\lambda_{1}^{3} \lambda_{2}+3 \lambda_{1}^{3} \lambda_{3}-1\right)\left(3 K_{2}-2 K_{3}\right)}{96 \Omega^{2}} A^{2} \\
& -\frac{K_{3}\left(\Omega^{2}-\alpha-\lambda_{1} \lambda_{2}-1\right)}{24 \Omega^{2}}, \\
M_{4}= & \frac{\left(2 \lambda_{1}^{5} \lambda_{2}+15 \lambda_{1}^{5} \lambda_{3}+2\right)\left(3 K_{2}-4 K_{3}\right)}{1152 \Omega^{2}} A^{4} \\
& -\frac{K_{3}\left(\lambda_{1}^{3} \lambda_{2}+3 \lambda_{1}^{3} \lambda_{3}-1\right)}{96 \Omega^{2}} A^{2}, \\
M_{5}= & \frac{\left(2 \lambda_{1}^{5} \lambda_{2}+15 \lambda_{1}^{5} \lambda_{3}+2\right)\left(3 K_{2}-4 K_{3}\right)}{1152 \Omega^{2}} A^{4} .
\end{aligned}
$$

And no secular terms require $M_{1}=0$; thus

$$
\begin{aligned}
& \frac{19\left(2 \lambda_{1}^{5} \lambda_{2}+15 \lambda_{1}^{5} \lambda_{3}+2\right)^{2}}{69120 \Omega^{2}} A^{8} \\
& +\frac{\left(\lambda_{1}^{3} \lambda_{2}+3 \lambda_{1}^{3} \lambda_{3}-1\right)\left(2 \lambda_{1}^{5} \lambda_{2}+15 \lambda_{1}^{5} \lambda_{3}+2\right)}{576 \Omega^{2}} A^{6} \\
& +\frac{\left(\lambda_{1}^{3} \lambda_{2}+3 \lambda_{1}^{3} \lambda_{3}-1\right)^{2}}{384 \Omega^{2}} A^{4}-\alpha=0 .
\end{aligned}
$$

By the same way, the second-order iteration solution can be obtained by substituting (12) into (17):

$$
\begin{aligned}
X_{2}= & \frac{M_{2}}{8 \Omega^{2}} \sin 3 \Omega \tau+\frac{M_{3}}{24 \Omega^{2}} \sin 5 \Omega \tau \\
& +\frac{M_{4}}{48 \Omega^{2}} \sin 7 \Omega \tau+\frac{M_{5}}{80 \Omega^{2}} \sin 9 \Omega \tau .
\end{aligned}
$$

By solving (15) and (19) simultaneously, we can obtain the frequency $\Omega$ and the auxiliary term $\alpha$. And the final solution $X$ can be uniformed by $X=X_{0}+X_{1}+X_{2}$.

\section{Results}

In order to verify the above method, the approximate solution by the new HPM was compared with the numerical solution solved by the Runge-Kutta method, as illustrated in Figure 2 with parameters: $V=1, \lambda_{1}=2, \lambda_{2}=10$, and $\lambda_{3}=20$. The results showed a good agreement.

Different parameters may lead to the different accuracy of the HPM solution. Table 1 showed the accuracy of HPM 


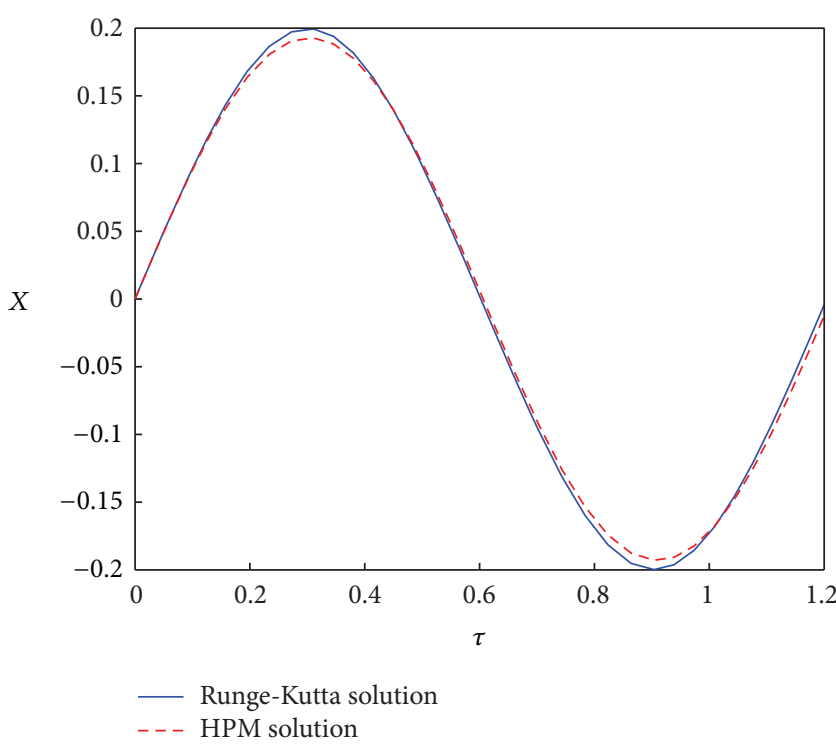

FIGURE 2: Comparison between the HPM solution and the numerical solution.

TABLE 1: Comparison between the HPM solution and the numerical solution.

\begin{tabular}{cccccc}
\hline$\lambda_{1}$ & $\lambda_{2}$ & $\lambda_{3}$ & $\Omega_{\mathrm{HPM}}$ & $\Omega_{\text {num }}$ & Error, \% \\
\hline 2 & 5 & 5 & 3.78959836 & 3.83806242 & 1.26272204 \\
2 & 5 & 10 & 4.02572209 & 4.04923784 & 0.58074509 \\
2 & 10 & 5 & 4.83451694 & 4.84579542 & 0.23274775 \\
2 & 10 & 20 & 5.18247480 & 5.22933694 & 0.89613923 \\
5 & 5 & 5 & 6.75089158 & 7.10746548 & 5.01689246 \\
5 & 5 & 10 & 7.36074401 & 7.8252235 & 5.93567059 \\
5 & 10 & 5 & 8.11755921 & 8.28280247 & 1.99501631 \\
5 & 10 & 20 & 9.15230023 & 9.42438670 & 2.88704696 \\
10 & 5 & 5 & 10.95651190 & 12.22175860 & 10.3524112 \\
10 & 5 & 10 & 12.09216730 & 13.21351496 & 8.48636917 \\
10 & 10 & 5 & 12.56277414 & 13.17320032 & 4.63384876 \\
10 & 10 & 20 & 14.66198678 & 15.63821607 & 6.24258730 \\
\hline
\end{tabular}

compared with the numerical solution in different $\lambda_{1}, \lambda_{2}$, and $\lambda_{3}$, and the results showed that, for different parameters, the HPM solutions were all in good agreement with the numerical solutions which can be almost equal to the exact solution.

\section{Conclusion}

It is desirable to obtain the solution of strong nonlinear equation arisen in polymer packaging system. In this paper, the homotopy perturbation method with an auxiliary term was applied, and the solution was obtained and compared with the Runge-Kutta method, showing good agreement. The results indicate that the HPM with an auxiliary term was suitable for solving the strong nonlinear vibration problems in packaging system. From the comparison results shown above, it can be concluded that, for the polymer packaging system with different parameters $\left(\lambda_{1}, \lambda_{2}\right.$, and $\left.\lambda_{3}\right)$, the HPM can be a vigorous method to obtain its frequency. What is more, different parameters will lead to different error value. And by roughly estimate, with the increase of $\lambda_{1}$, the HPM solution's relative error increases; with the increase of $\lambda_{2}$ and $\lambda_{3}$, the HPM solution's relative error decreases. Thus, the future research can focus on the contribution of different $\lambda_{1}, \lambda_{2}$, and $\lambda_{3}$ to the HPM's accuracy and to what range of $\lambda_{1}, \lambda_{2}$, and $\lambda_{3}$ values the HPM can be applied.

\section{Parameter List}

$\begin{array}{ll}x: & \text { The displacement of the product } \\ & \text { while dropping, } \mathrm{m} \\ m: & \text { The mass of the packaged product, } \mathrm{kg} \\ \beta_{1} \text { and } \beta_{2}: & \text { The displacement impendence, } \mathrm{m}^{-1} \\ \beta_{3}, \beta_{4}, \text { and } \beta_{5}: & \text { The elasticity, } \mathrm{N} \\ g: & \text { The acceleration of gravity, } \mathrm{m} / \mathrm{s}^{-2} \\ h: & \text { The dropping height, } \mathrm{m} \\ T_{0}, L, \text { and } \lambda_{\mathrm{i}}: & \text { Nondimensionalized parameters } \\ X: & \text { Nondimensionalized displacement } \\ \tau: & \text { Nondimensionalized time } \\ V: & \text { Nondimensionalized velocity } \\ A: & \text { Nondimensionalized displacement } \\ \Omega: & \text { amplitude } \\ K_{\mathrm{i}} \text { and } M_{\mathrm{i}}: & \text { Nondimensionalized frequency } \\ & \text { Parameters used to simplify the } \\ \alpha: & \text { expression }\end{array}$

\section{Acknowledgments}

This work was supported by the National Natural Science Foundation of China (Grant no. 51205167), Research Fund of young scholars for the Doctoral Program of Higher Education of China (Grant no. 20120093120014), and Fundamental Research Funds for the Central Universities (Grant no. JUSRP51302A).

\section{References}

[1] J. Wang, Z. W. Wang, L. X. Lu, Y. Zhu, and Y. G. Wang, "Three-dimensional shock spectrum of critical component for nonlinear packaging system," Shock and Vibration, vol. 18, no. 3, pp. 437-445, 2011.

[2] J. Wang, J. H. Jiang, L. X. Lu, and Z. W. Wang, "Dropping damage evaluation for a tangent nonlinear system with a critical component," Computers and Mathematics with Applications, vol. 61, no. 8, pp. 1979-1982, 2011.

[3] J. H. Jiang and Z. W. Wang, "Dropping damage boundary curves for cubic and hyperbolic tangent packaging systems based on key component," Packaging Technology and Science, vol. 25, no. 7, pp. 397-411, 2012.

[4] J. D. Cole, Perturbation Methods in Applied Mathematics, Blaisdell Publishing, 1968.

[5] M. G. Sakar, F. Erdogan, and A. Ylldırım, "Variational iteration method for the time-fractional Fornberg-Whitham equation," Computers \& Mathematics with Applications, vol. 63, no. 9, pp. 1382-1388, 2012. 
[6] S. Liao, "On the homotopy analysis method for nonlinear problems," Applied Mathematics and Computation, vol. 147, no. 2, pp. 499-513, 2004.

[7] J. H. He, "Some asymptotic methods for strongly nonlinear equations," International Journal of Modern Physics B, vol. 20, no. 10, pp. 1141-1199, 2006.

[8] E. Hetmaniok, I. Nowak, D. Słota, and R. Wituła, "A study of the convergence of and error estimation for the homotopy perturbation method for the Volterra-Fredholm integral equations," Applied Mathematics Letters, vol. 26, no. 1, pp. 165-169, 2013.

[9] J. Singh, D. Kumar, and A. Kılıçman, "Homotopy perturbation method for fractional gas dynamics equation using sumudu transform," Abstract and Applied Analysis, vol. 2013, Article ID 934060, 8 pages, 2013.

[10] E. Hetmaniok, I. Nowak, D. Słota, and R. Wituła, "Application of the homotopy perturbation method for the solution of inverse heat conduction problem," International Communications in Heat and Mass Transfer, vol. 39, no. 1, pp. 30-35, 2012.

[11] S. T. Mohyud-Din, A. Yildırım, and M. Inc, "Coupling of homotopy perturbation and modified Lindstedt-Poincaré methods for traveling wave solutions of the nonlinear Klein-Gordon equation," Journal of King Saud University, vol. 24, no. 2, pp. 187191, 2012.

[12] H. Jafari and C. Khalique, "Homotopy perturbation and variational iteration methods for solving fuzzy differential equations," Communications in Fractional Calculus, vol. 3, no. 1, pp. 38-48, 2012.

[13] M. Javidi and M. Raji, "Combination of Laplace transform and homotopy perturbation method to solve the parabolic partial differential equations," Communications in Fractional Calculus, vol. 3, no. 1, pp. 10-19, 2012.

[14] F. Wang, W. Li, and H. Zhang, "A new extended homotopy perturbation method for nonlinear differential equations," Mathematical and Computer Modelling, vol. 55, no. 3-4, pp. 1471-1477, 2012.

[15] Q. M. Al-Mdallal, M. I. Syam, and P. D. Ariel, "Extended homotopy perturbation method and the axisymmetric flow past a porous stretching sheet," International Journal for Numerical Methods in Fluids, vol. 69, no. 5, pp. 909-925, 2012.

[16] S. L. Mei, "Construction of target controllable image segmentation model based on homotopy perturbation technology," Abstract and Applied Analysis, vol. 2013, Article ID 131207, 8 pages, 2013.

[17] D. Vogt, "On approximate analytical solutions of differential equations in enzyme kinetics using homotopy perturbation method," Journal of Mathematical Chemistry, vol. 51, no. 3, pp. 826-842, 2013.

[18] Y. Nawaz, "Variational iteration method and homotopy perturbation method for fourth-order fractional integro-differential equations," Computers \& Mathematics with Applications, vol. 61, no. 8, pp. 2330-2341, 2011.

[19] M. A. Noor, "Some iterative methods for solving nonlinear equations using homotopy perturbation method," International Journal of Computer Mathematics, vol. 87, no. 1-3, pp. 141-149, 2010.

[20] J. H. He, "Homotopy perturbation method with an auxiliary term," Abstract and Applied Analysis, vol. 2012, Article ID 857612, 7 pages, 2012.

[21] Q. Hu and Z. F. Tong, "Nonlinear elastic models of foamed plastics cushions for packages," Journal of Vibration and Shock, vol. 9, no. 2, pp. 9-13, 1990 (Chinese). 


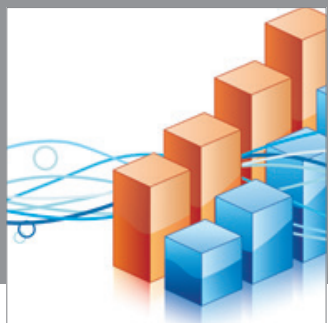

Advances in

Operations Research

mansans

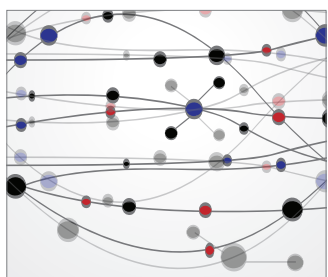

The Scientific World Journal
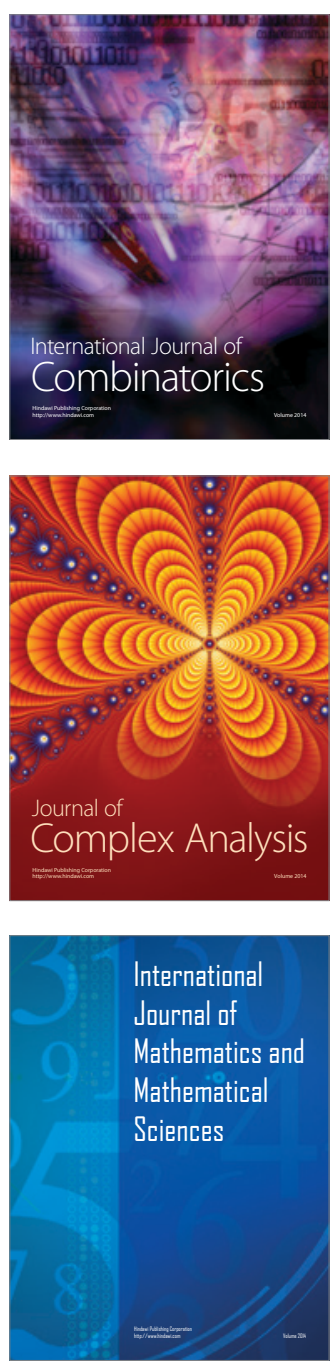
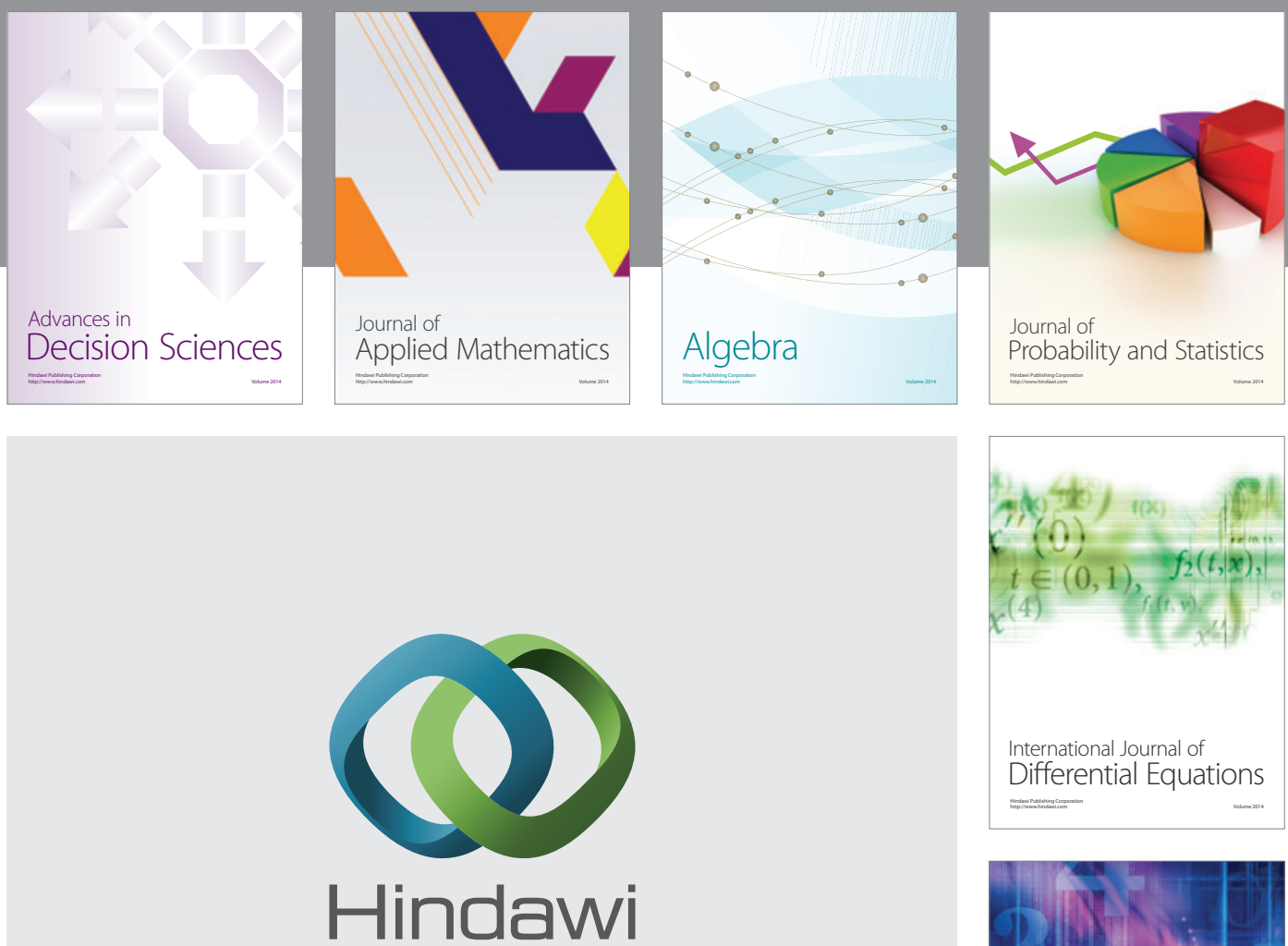

Submit your manuscripts at http://www.hindawi.com
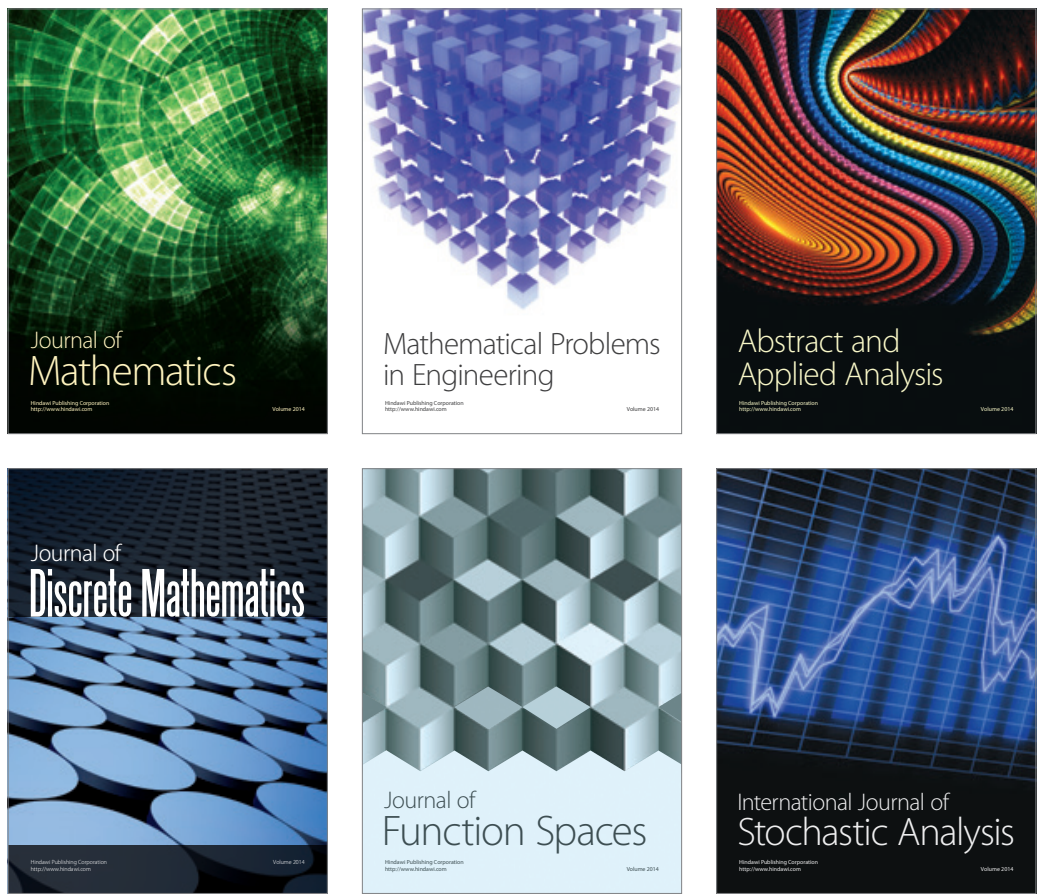

Journal of

Function Spaces

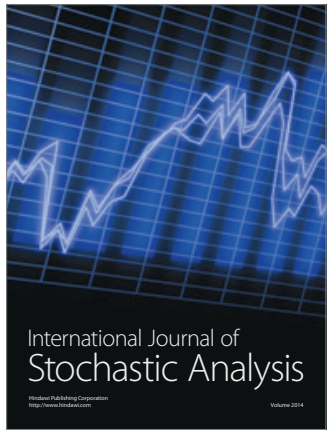

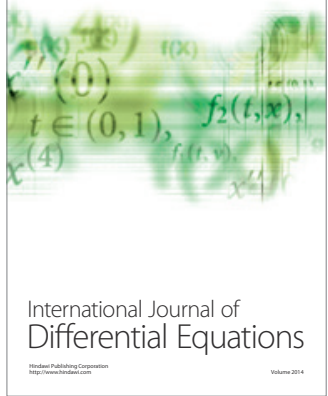
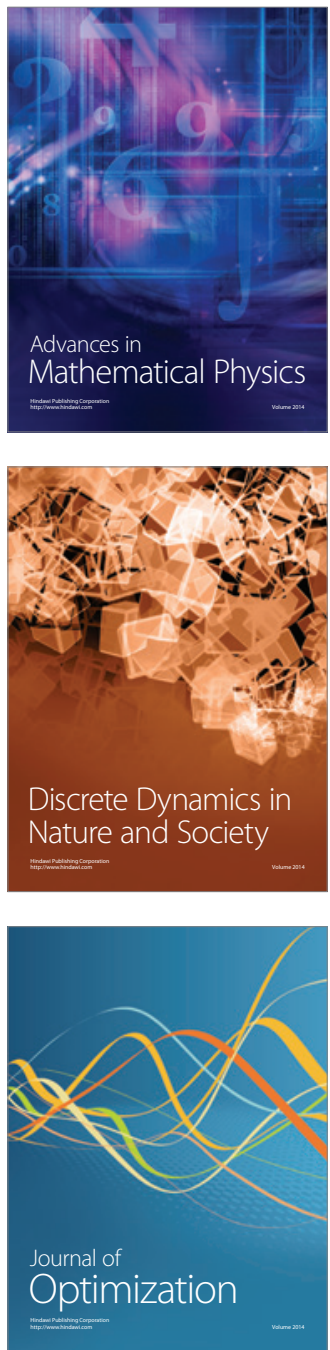